TRANSACTIONS OF THE

AMERICAN MATHEMATICAL SOCIETY

Volume 364, Number 4, April 2012, Pages 2109-2128

S 0002-9947(2011)05523-6

Article electronically published on November 9, 2011

\title{
ON THE COALESCENCE TIME OF REVERSIBLE RANDOM WALKS
}

\author{
ROBERTO IMBUZEIRO OLIVEIRA
}

\begin{abstract}
Consider a system of coalescing random walks where each individual performs a random walk over a finite graph $\mathbf{G}$ or (more generally) evolves according to some reversible Markov chain generator $Q$. Let $C$ be the first time at which all walkers have coalesced into a single cluster. $C$ is closely related to the consensus time of the voter model for this $\mathbf{G}$ or $Q$.

We prove that the expected value of $C$ is at most a constant multiple of the largest hitting time of an element in the state space. This solves a problem posed by Aldous and Fill and gives sharp bounds in many examples, including all vertex-transitive graphs. We also obtain results on the expected time until only $k \geq 2$ clusters remain. Our proof tools include a new exponential inequality for the meeting time of a reversible Markov chain and a deterministic trajectory, which we believe to be of independent interest.
\end{abstract}

\section{INTRODUCTION}

Consider a system of continuous-time random walks on a finite connected graph $\mathbf{G}$, with a walker starting from each vertex of $\mathbf{G}$. Let the walkers evolve independently, except that any two that occupy the same vertex of $\mathbf{G}$ at a given time coalesce into one (this is made precise in Section 3.2).

As time goes by, larger and larger coalesced clusters emerge, until at a certain random time $C$ only one cluster remains. The question we address here is: how large can $C$ be in terms of other parameters of $\mathbf{G}$ ? This is a natural question which has implications for the so-called voter model on $\mathbf{G}$, discussed in Section 1.1 below.

It is instructive to consider what happens in the simple case of $\mathbf{G}=\mathbf{K}_{n}$, the complete graph on $n$ vertices. An explicit calculation [2, Chapter 14, Sec. 3.3] shows that, for large $n$,

$$
\frac{C}{n} \approx \sum_{i=1}^{+\infty} \frac{Z_{i}}{i(i+1)}, \text { with }\left\{Z_{i}\right\}_{i \geq 1} \text { i.i.d. exponential r.v.'s with mean } 1 .
$$

In particular, $\mathbb{E}[C] \sim n$ as $n \rightarrow+\infty$. What is remarkable about this is that any two of the walkers will take an expected time $\sim n / 2$ to meet and coalesce. The fact that we are dealing with an unbounded number of particles only increases the expected time by a constant factor.

Received by the editors September 3, 2010 and, in revised form, September 9, 2010.

2010 Mathematics Subject Classification. Primary 60J27, 60K35; Secondary 60C05.

Key words and phrases. Coalescing random walks, voter model, hitting time.

The author's work was supported by Bolsa de Produtividade em Pesquisa and by a Pronex grant from CNPq, Brazil.

(C)2011 American Mathematical Society Reverts to public domain 28 years from publication 
It is natural to ask what happens in more general graphs. This is closely related to the following problem, which was posed by Aldous and Fill in the mid-nineties.

Problem 1.1 (Open Problem 13, Chapter 14 of [2]). Prove that there exists a universal constant $K>0$ such that, for any graph $\mathbf{G}$, the expected value of $C$ satisfies

$$
\mathbb{E}[C] \leq K \mathrm{~T}_{\text {hit }}^{\mathbf{G}},
$$

irrespective of initial conditions, where $\mathrm{T}_{\text {hit }}^{\mathbf{G}}$ is the maximum expected hitting time of a vertex in $\mathbf{G}$.

To see how this relates to our previous discussion, let $T_{\text {meet }}^{\mathbf{G}}$ denote the maximum expected meeting time of two walkers over $\mathbf{G}$. If $\mathbf{G}$ is vertex-transitive, Proposition 5 in [2, Chapter 14] implies $\mathrm{T}_{\text {meet }}^{\mathbf{G}}=\mathrm{T}_{\text {hit }}^{\mathbf{G}} / 2$. Therefore, if Problem 1.1 has a positive solution, all vertex-transitive graphs are like $\mathbf{K}_{n}$ in that $\mathbb{E}[C]$ is at most a universal constant factor away from $\mathrm{T}_{\text {meet }}^{\mathbf{G}}$. A similar conclusion holds for other families of graphs where $\mathrm{T}_{\text {meet }}^{\mathbf{G}}=\Theta\left(\mathrm{T}_{\text {hit }}^{\mathbf{G}}\right)$ (e.g. all regular graphs with $\mathrm{T}_{\text {hit }}^{\mathbf{G}}=O(n)$, where $n$ is the number of vertices in $\mathbf{G}$ ). For more general graphs it is still true that $\mathrm{T}_{\text {meet }}^{\mathrm{G}} \leq \mathrm{T}_{\text {hit }}^{\mathrm{G}}$, as proven in the aforementioned proposition (see also [1]), and the problem may be viewed as a strengthening of this fact.

Remark 1.2. It is not true that $\mathbb{E}[C] \leq K \mathrm{~T}_{\text {meet }}^{\mathrm{G}}$ or $\mathrm{T}_{\text {hit }}^{\mathrm{G}} \leq K \mathrm{~T}_{\text {meet }}^{\mathrm{G}}$ with $K$ universal. For instance, in $n$-vertex star graphs, one can show that $\mathrm{T}_{\text {meet }}^{\mathbf{G}}=\Theta(1), \mathbb{E}[C]=$ $\Theta(\ln n)$ and $\mathrm{T}_{\text {hit }}^{\mathbf{G}}=\Theta(n)$ as $n \rightarrow+\infty$.

To the best of our knowledge, Problem 1.1 has remained open up to now. The best known bound of this sort has an extra $\ln |\mathbf{V}|$ factor; see Proposition 11 in 2, Chapter 14]. Our main goal in this paper is to give a solution to Problem 1.1 in the more general setting of reversible Markov chains.

Assume that $Q$ is the generator of a reversible, irreducible, continuous-time Markov chain over a finite set $\mathbf{V}$. Given $v \in \mathbf{V}$, let $H_{v}$ be the hitting time of $v$, i.e. the first time at which a trajectory of $Q$ hits $v$. We define the following parameter of the chain:

$$
\mathrm{T}_{\text {hit }}^{Q} \equiv \max _{v, w \in \mathbf{V}} \mathbb{E}_{w}\left[H_{v}\right]=\text { largest expected hitting time for } Q .
$$

Define a system of coalescing random walks as in the case of graphs, with the difference that each walker now evolves over $\mathbf{V}$ according to $Q$. The following Theorem solves Problem 1.1.

Theorem 1.3. There exists a universal constant $K>0$ such that, with $Q$ as above, for any $n \in \mathbb{N} \backslash\{0\}$ and for any $x^{(n)}=(x(1), \ldots, x(n)) \in \mathbf{V}^{n}$,

$$
\mathbb{E}_{x^{(n)}}[C] \leq K \mathrm{~T}_{\text {hit }}^{Q} .
$$

Remark 1.4. Here $x^{(n)}$ is an initial condition with $n$ arbitrary. In particular, there may be more or less than one walker at each site $v \in \mathbf{V}$ at the beginning of the process. Allowing for arbitrary initial conditions is convenient for our proofs but does not really change the results.

We also prove a stronger result. Let $C_{k}$ denote the first time at which there are at most $k$ clusters of coalesced walkers $(k \geq 1)$. Notice that $C_{1}=C$ with this definition. 
Theorem 1.5. There exists a universal constant $K_{1}>0$ such that, in the same setting as Theorem 1.3 ,

$$
\forall k \in \mathbb{N} \backslash\{0\}, \mathbb{E}_{x^{(n)}}\left[C_{k}\right] \leq K_{1}\left(\frac{\mathrm{T}_{\text {hit }}^{Q}}{k}+\mathrm{T}_{\text {mix }}^{Q}\right),
$$

where $\mathrm{T}_{\mathrm{mix}}^{Q}$ is the mixing time of $Q$ (see Section 2.2 for a definition).

The dependence on $k$ in this theorem is essentially best possible, as $\mathbb{E}_{x^{(n)}}\left[C_{k}\right] \sim$ $\mathrm{T}_{\text {hit }}^{Q} / k$ for large complete graphs. The case $k=1$ gives back Theorem [1.3, as $\mathrm{T}_{\text {mix }}^{Q} \leq c \mathrm{~T}_{\text {hit }}^{Q}$ for some universal $c>0$ [2, Chapter 4]. We will nevertheless prove Theorem 1.3 first and then show how its proof can be modified to obtain Theorem 1.5 .

One justification for proving this second result is that it is helpful in approximating the distribution of $C$. We are in the process of writing a paper where we show that, if $Q$ is transitive and $\mathrm{T}_{\text {mix }}^{Q} \ll \mathrm{T}_{\text {hit }}^{Q}$, then

$$
\text { law of } \left.\frac{C}{\mathrm{~T}_{\text {hit }}^{Q}} \approx \sum_{i=1}^{+\infty} \frac{Z_{i}}{i(i+1)} \text {, as for } \mathbf{K}_{n} \text { (cf. (1.1) }\right) \text {. }
$$

In particular, $\mathbb{E}[C] \sim \mathrm{T}_{\text {hit }}^{Q}$. This was previously known only for discrete tori $\mathbb{Z}_{L}^{d}$ with $L \gg 1$ in $d \geq 2$ dimensions, due to Cox's paper 44 An important step in both our proof and Cox's argument is that $\mathbb{E}\left[C_{k}\right] \ll \mathrm{T}_{\text {hit }}^{Q}$ if $k \gg 1$. Cox proves this in [4, Section 4] via a simple renormalization argument which is very specific for discrete tori, whereas we use Theorem 1.5 for the same purpose.

1.1. Application to the voter model. We now sketch the connection between our results and the voter model [6, 2] on a graph $\mathbf{G}$ (this could be generalized to an arbitrary generator $Q$, but we will not do this here). The state of the process at a given time $t$ is a function

$$
\eta_{t}: V(\mathbf{G}) \rightarrow \mathcal{O}
$$

where $V(\mathbf{G})$ is the vertex set of $\mathbf{G}$ and $\mathcal{O}$ is a fixed set of possible opinions. The evolution of the process is as follows. Each vertex $v \in V(\mathbf{G})$ "wakes up" at rate 1 . When that happens at a time $t>0, v$ chooses one of its neighbors $w$ uniformly at random and updates its value of $\eta_{t}(v)$ to $w$ 's opinion $\eta_{t_{-}}(w)$; all other opinions stay the same.

A classical duality result (see e.g. 6, Chapter 5] or 2, Chapter 14]) relates the state of the process at a given time to a system of coalescing random walks on $\mathbf{G}$ moving backwards in time. In particular, the consensus time for the voter model i.e. the least time at which all vertices of $\mathbf{G}$ have the same opinion - is dominated by the coalescence time $C$ from the initial state with all vertices occupied. This implies the following corollary of Theorem 1.3 .

Corollary 1.6. There exists a universal constant $K>0$ such that, for any graph $\mathbf{G}$ and any set $\mathcal{O}$, the expected value of the consensus time of the voter model defined in terms of $\mathbf{G}$ and $\mathcal{O}$, started from an arbitrary initial state, is bounded by $K \mathrm{~T}_{\text {hit }}^{\mathbf{G}}$.

\footnotetext{
${ }^{1}$ Transitivity can be dropped at the cost of making stronger assumptions on $Q$ and using a different normalization factor.
} 
Proposition 5 in [2, Chapter 14] shows that the corollary is tight up to the value of $K$ for vertex-transitive $\mathbf{G}$, at least when the initial conditions are iid uniform over $\{-1,+1\}$ (say); we omit the details.

1.2. Main proof ideas. Let us give an outline of the (elementary) proof of Theorem 1.3. the proof of Theorem 1.5 is quite similar. For clarity, we first present an oversimplified account and then explain how one can avoid the oversimplifications.

We consider $n$ walkers $\left(X_{t}(a)\right)_{t \geq 0}$ evolving according to $Q$, labeled with numbers $a=1, \ldots, n$ and respective initial states $x^{(1)}, \ldots, x^{(n)}$. Instead of having walkers coalesce, we will assume that at any given time each walker is either alive or dead. All walkers are alive in the beginning, and walker $\# a$ is killed at the first time $t$ when an alive walker with label $b<a$ satisfies $X_{t}(a)=X_{t}(b)$ (this is made precise in Section 3.3). The number of walkers that are alive at time $t$ in this process is precisely the number of clusters in the coalescing random walks process, and $C$ is the first time at which only walker $\# 1$ is still alive. This implies that

$$
\mathbb{P}(C>t) \leq \sum_{a=2}^{n} \mathbb{P}(a \text { alive at time } t) .
$$

We now make the following oversimplification:

Oversimplification $\# 1$ : walker $\# a$ dies at the first time when $X_{t}(a)=X_{t}(b)$ for some $b<a$.

The reason why this is an oversimplification is that a walker \#b may have died before meeting walker \#a. We ignore this for the moment and write

$$
\mathbb{P}(a \text { alive at time } t) \leq \mathbb{P}\left(\bigcap_{b=1}^{a-1}\left\{\forall 0 \leq s \leq t, X_{s}(a) \neq X_{s}(b)\right\}\right)
$$

In order to simplify the RHS, we notice that the trajectories $\left(X_{t}(u)\right)_{t \geq 0}$ of walkers $\# u, 1 \leq u \leq a$, are independent realizations of $Q$. Conditioning on $X_{s}(a)=h_{s}$, $s \geq 0$, makes the events in the RHS independent, and we deduce that

$$
\mathbb{P}\left(a \text { alive at time } t \mid\left(X_{s}(a)\right)_{s \geq 0}=\left(h_{s}\right)_{s \geq 0}\right) \leq \prod_{b=1}^{a-1} \mathbb{P}\left(\forall 0 \leq s \leq t, X_{s}(b) \neq h_{s}\right) .
$$

We now make another oversimplification.

Oversimplification \#2: $\left(X_{t}(b)\right)_{t \geq 0}$ is started from the stationary distribution for all $b$.

This allows us to use the following lemma, which we believe to be new (and of independent interest).

Lemma 1.7 (Meeting Time Lemma; proven in Section [5.2). Let $\left(X_{t}\right)_{t \geq 0}$ be a realization of $Q$ starting from the stationary distribution $\pi$. Then there exist $v \in \mathbf{V}$ and a quasistationary distribution $\mathbf{q}_{v}$ for $\mathbf{V} \backslash\{v\}$ such that for any deterministic path $h \in \mathbb{D}([0,+\infty), \mathbf{V})$, we have

$$
\forall t \geq 0, \mathbb{P}_{\pi}\left(\forall 0 \leq s \leq t, X_{s} \neq h_{s}\right) \leq \mathbb{P}_{\mathrm{q}_{v}}\left(H_{v}>t\right)=\exp \left(-\frac{t}{\mathbb{E}_{\mathrm{q}_{v}}\left[H_{v}\right]}\right) .
$$

Remark 1.8. The proof of Lemma 1.7 shows that we may take $v \in h([0,+\infty))$. This is a well-known result if $h \equiv v$ [2, Chapter 3, Section 6.5]. An application of this lemma to so-called cat-and-mouse games is sketched in the final section. 
Notice that $\mathbb{E}_{\mathbf{q}_{v}}\left[H_{v}\right] \leq \mathrm{T}_{\text {hit }}^{Q}$, so:

$$
\mathbb{P}\left(a \text { alive at time } t \mid\left(X_{s}(i)\right)_{s \geq 0}=\left(h_{s}\right)_{s \geq 0}\right) \leq e^{-\frac{(a-1) t}{\mathrm{~T}_{\text {hit }}^{Q}}} .
$$

This shows that

$$
\mathbb{P}(C>t) \leq \sum_{a=2}^{n} e^{-\frac{(a-1) t}{\mathrm{~T}_{\mathrm{hit}}^{Q}}} .
$$

If one takes $t=(\ln 2+c) \mathrm{T}_{\text {hit }}^{Q}$, the RHS becomes

$$
\mathbb{P}\left(C>(\ln 2+c) \mathrm{T}_{\text {hit }}^{Q}\right) \leq \sum_{a=2}^{n} 2^{-a+1} e^{-(a-1) c} \leq e^{-c},
$$

and this gives $\mathbb{E}[C] \leq(\ln 2+1) \mathrm{T}_{\text {hit }}^{Q}$.

Of course, this is not a proof of Theorem 1.3 because of the oversimplifications. Our way out of this is to introduce a process where at any given time there is a list of allowed killings. At any time $t$ there will be a set $\mathcal{A}_{t}$ so that walker $\# b$ may kill walker $\# a$ at time $t$ only if $b<a$ and $(b, a) \in \mathcal{A}_{t}$ (cf. Section 3.4). The salient characteristics of this process are:

1. For any choice of $\mathcal{A}=\left(\mathcal{A}_{t}\right)_{t \geq 0}$, the set of alive walkers in the process defined via $\mathcal{A}$ dominates the corresponding set in the process without $\mathcal{A}$ (see Proposition 3.4).

2. A judicious choice of $\mathcal{A}$ will ensure that for each $a$ there will be a large enough time interval where a large number of walkers will be available to kill walker \#a. Moreover, many of those walkers will be stationary.

Item 1 allows us to consider the process with a list of allowed killings instead of the original process in order to obtain upper bounds. Item 2 will mean that we may apply the Meeting Time Lemma to at least some of the walkers with indices $b<a$, in some time intervals. These two ingredients will allow us to "fix" the oversimplified proof just presented.

1.3. Organization. The remainder of the paper is organized as follows. Section 2 introduces our notation and recalls some basic concepts. Section 3 defines the main processes we consider in the paper. Section 4 presents the proofs of the two theorems, and Section 5 presents the proof of Lemma 1.7. Some final comments are presented in the last section.

\section{Preliminaries}

In what follows we recall some basic material while also fixing notation.

2.1. Basic notation. $\mathbb{N}$ is the set of non-negative integers. Given $n \in \mathbb{N} \backslash\{0\}$, we set $[n] \equiv\{1,2, \ldots, n\}$.

We will often speak of universal constants. These are numbers that are independent of any other object or parameter under consideration, be it a Markov chain, the initial state of a process under consideration or anything else.

The cardinality of a finite set $S$ is denoted by $|S|$, and $2^{S}$ represents the power set of $S$ (i.e. the set whose elements are the subsets of $S$ ). The set of all probability measures over $S$ will be denoted by $M_{1}(S)$. $\mathbb{R}^{S}$ denotes the space of all functions $f: S \rightarrow \mathbb{R}$, or equivalently of all (column) vectors with entries indexed by $S$. Linear operators acting on $\mathbb{R}^{S}$ correspond to matrices with rows and columns indexed by 
the elements of $S$. If $A$ is some matrix of this sort, $\|A\|_{\text {op }}$ is the operator norm of $A$. If $A$ is symmetric, we let $\lambda_{\min }(A), \lambda_{\max }(A)$ denote the minimum and maximum eigenvalues of $A$ (respectively).

Given a finite set $F \neq \emptyset$, a function $\omega:[0,+\infty) \rightarrow F$ is said to be càdlàg if there exist $t_{0}=0<t_{1}<t_{2}<\cdots<t_{n}<\cdots \nearrow{ }+\infty$ with $\omega$ constant over each interval $\left[t_{i-1}, t_{i}\right) . \mathbb{D}([0,+\infty), F)$ is the set of all such càdlàg functions, with the $\sigma$-field generated by the projections " $\omega \mapsto \omega(t)$ " ( $t \geq 0)$.

2.2. Markov chain basics. Let $\mathbf{V}$ be a finite, non-empty set. A matrix $Q$ (with rows and columns labelled by $\mathbf{V}$ ) which acts on $\mathbb{R}^{\mathbf{V}}$ in the following way:

$$
Q: f(\cdot) \in \mathbb{R}^{\mathbf{V}} \mapsto \sum_{x \in \mathbf{V}, x \neq} q(\cdot, x)(f(\cdot)-f(x)), \text { with } q(\cdot, \cdot \cdot) \geq 0,
$$

defines a unique continuous-time Markov chain on V. More precisely, there exists a unique family of measures $\left\{\mathbb{P}_{x}\right\}_{x \in \mathbf{V}}$ over $\mathbb{D}([0,+\infty), \mathbf{V})$ (with the $\sigma$-field generated by finite-dimensional projections) such that, letting

$$
X_{t}: \omega \in \mathbb{D}([0,+\infty), \mathbf{V}) \mapsto X_{t}(\omega) \equiv \omega(t) \in \mathbf{V}
$$

and $\mathcal{F}_{t} \equiv \sigma\left(X_{s}: s \leq t\right)$, we have $\mathbb{P}_{x}\left(X_{0}=x\right)=1$ and

$$
\mathbb{P}_{x}\left(X_{t+s}=y \mid \mathcal{F}_{s}\right)=\text { the entry of } e^{-t Q} \text { labelled by }\left(X_{t}, y\right)(t, s \geq 0, y \in \mathbf{V}) .
$$

$Q$ is said to be the generator of the Markov chain and the numbers $q(x, y)(x, y \in \mathbf{V}$, $x \neq y)$ are the transition rates. We let $\mathbb{E}_{x}[\cdot]$ denote expectation with respect to $\mathbb{P}_{x}$.

We also define

$$
\mathbb{P}_{\mu}=\sum_{x \in \mathbf{V}} \mu(x) \mathbb{P}_{x}, \quad\left(\mu \in M_{1}(\mathbf{V})\right)
$$

which we interpret in the customary way as describing the law of the chain given by $Q$ from a random initial state with law $\mu . \mathbb{E}_{\mu}[\cdot]$ is the corresponding expectation symbol.

We will always assume that $Q$ is irreducible, meaning that for all $A \subset \mathbf{V}$ with $A, \mathbf{V} \backslash A \neq \emptyset$ there exist $a \in A$ and $b \in \mathbf{V} \backslash A$ with $q(a, b) \neq 0$. In this case there exists a unique probability measure $\pi \in M_{1}(\mathbf{V})$ which is stationary in the sense that $\mathbb{P}_{\pi}\left(X_{t}=\cdot\right)=\pi(\cdot)$ for all $t \geq 0$. Moreover, we have that

$$
\forall x, y \in \mathbf{V}, \lim _{t \rightarrow+\infty} \mathbb{P}_{x}\left(X_{t}=y\right)=\pi(y) .
$$

The mixing time of $Q$ measures the speed of this convergence:

$$
\mathrm{T}_{\text {mix }}^{Q} \equiv \inf \left\{t \geq 0: \forall x \in \mathbf{V}, \max _{S \subset \mathbf{V}}\left|\mathbb{P}_{x}\left(X_{t} \in S\right)-\pi(S)\right| \leq 1 / 4\right\} .
$$

Finally, we will also assume that $Q$ is reversible with respect to $\pi$, which means that $\pi(x) q(x, y)=\pi(y) q(y, x)$ for all distinct $x, y \in \mathbf{V}$. This is the same as requiring that the matrix $\Pi^{1 / 2} Q \Pi^{-1 / 2}$ is symmetric, where $\Pi$ is diagonal and has the values $\pi(v), v \in \mathbf{V}$ on the diagonal.

\section{Processes With multiple Random Walks}

Here we define the main processes that we will be concerned with, all of which involve $n$ random walkers for some integer $n \in \mathbb{N} \backslash\{0,1\}$. We will assume that $Q$ and $\left\{\mathbb{P}_{x}\right\}_{x \in \mathbf{V}}$ are as defined in Section 2.2 
3.1. Independent random walks. We first define a process made out of $n$ independent realizations of the Markov chain with generator $Q$. More specifically, given

$$
x^{(n)}=(x(1), x(2), \ldots, x(n)) \in \mathbf{V}^{n},
$$

we let $\mathbb{P}_{x^{(n)}}$ denote the distribution on $\mathbb{D}\left([0,+\infty), \mathbf{V}^{n}\right)$ corresponding to $n$ independent trajectories of $Q$,

$$
\left(X_{t}^{(n)}\right)_{t \geq 0} \equiv\left(X_{t}(a): a \in[n]\right)_{t \geq 0},
$$

with each $\left(X_{t}(a)\right)_{t \geq 0}$ started from $x(a)$. That is, the joint law of $\left\{\left(X_{t}(a)\right)_{t \geq 0}\right\}_{a \in[n]}$ is the product measure

$$
\mathbb{P}_{x(1)} \times \mathbb{P}_{x(2)} \times \cdots \times \mathbb{P}_{x(n)}
$$

Notice that our notation $\mathbb{P}_{x^{(n)}}$ does not refer explicitly to the fact that this is a process on $\mathbf{V}^{n}$, as opposed to the process over $\mathbf{V}$ defined in the previous subsection. This distinction should be clear from context and from the fact that we write all $x^{(n)} \in \mathbf{V}^{n}$ with an " $(n)$ " superscript. The independent random walks process is also a Markov chain: for $x^{(n)}=(x(1), \ldots, x(n))$ and $y^{(n)}=(y(1), \ldots, y(n))$ distinct, the transition rate from $x^{(n)}$ to $y^{(n)}$ is

$$
q^{(n)}\left(x^{(n)}, y^{(n)}\right) \equiv \begin{cases}q(x(i), y(i)) & \text { if } x(i) \neq y(i) \text { and } \\ 0 & x(j)=y(j) \text { for all } j \in[n] \backslash\{i\} \\ 0 & \text { otherwise. }\end{cases}
$$

3.2. Coalescing random walks. For our purposes, it is convenient to define this process, denoted by

$$
\left(\mathrm{Co}_{t}^{(n)}\right)_{t \geq 0} \equiv\left(\mathrm{Co}_{t}(a): a \in[n]\right)_{t \geq 0},
$$

as a deterministic function of the independent random walks process. The idea is that once a walker meets another walker with smaller index, it starts following the trajectory of the latter. That is, consider a realization of $\mathbb{P}_{x^{(n)}}$ as in (3.1). First define

$$
\mathrm{Co}_{t}(1) \equiv X_{t}(1) \quad(t \geq 0)
$$

Given $a \in[n] \backslash\{1\}$, assume inductively that $\left(\mathrm{Co}_{t}(b)\right)_{t \geq 0}$ has been defined for $1 \leq$ $b<a$. Since $Q$ is irreducible, there a.s. is a first time $\tau_{a}$ at which $X_{t}(a)=\mathrm{Co}_{t}(b)$ for some $b \in[a-1]$. More precisely, define

$$
\tau_{a} \equiv \inf \left\{t \geq 0: \exists 1 \leq b<a, X_{t}(a)=\mathrm{Co}_{t}(b)\right\}
$$

and

and then set:

$$
B_{a} \equiv \min \left\{b \in[a-1]: X_{\tau_{a}}(a)=X_{\tau_{a}}(b)\right\},
$$

$$
\mathrm{Co}_{t}(a) \equiv\left\{\begin{array}{ll}
X_{t}(a), & 0 \leq t<\tau_{a}, \\
\mathrm{Co}_{t}\left(B_{a}\right), & t \geq \tau_{a},
\end{array} \text { for each } t \geq 0 .\right.
$$

One can show that the law of $\left(\mathrm{Co}_{t}^{(n)}\right)_{t \geq 0}$ is invariant under permutations of the $x(i)$. We also define the set

$$
S_{t} \equiv\left\{v \in \mathbf{V}: \exists a \in[n], \mathrm{Co}_{t}(a)=v\right\}
$$

as the set of occupied sites in this process. Our definition of $\left(S_{t}\right)_{t \geq 0}$ coincides with the more traditional coalescing random walks process defined in e.g. 4. We also 
set

and $C \equiv C_{1}$.

$$
C_{k} \equiv \inf \left\{t \geq 0:\left|S_{t}\right| \leq k\right\} \quad(k \in \mathbb{N} \backslash\{0\})
$$

Remark 3.1. We note that this process makes sense even if $x^{(n)}$ contains repeats, i.e. if there exist $i \neq j$ with $x(i)=x(j)$.

3.3. Random walks with killings. Let $\partial \notin V$ be a "coffin state". We define a new process

$$
\left(Y_{t}^{(n)}\right)_{t \geq 0} \equiv\left(Y_{t}(a): a \in[n]\right)_{t \geq 0} .
$$

The new idea is that a walker with index $a$ will be killed by a walker of index $b<a$ occupying the same site. More precisely, we first define

$$
Y_{t}(1) \equiv X_{t}(1) \quad(t \geq 0) .
$$

Given $a \in[n] \backslash\{1\}$, assume inductively that $\left(Y_{t}(b)\right)_{t \geq 0}$ has been defined for $1 \leq b<$ a. Define

$$
\tau_{a} \equiv \inf \left\{t \geq 0: \exists 1 \leq b<a, X_{t}(a)=Y_{t}(b)\right\}
$$

and set

$$
Y_{t}(a) \equiv\left\{\begin{array}{ll}
X_{t}(a), & 0 \leq t<\tau_{a}, \\
\partial, & t \geq \tau_{a},
\end{array} \quad \text { for each } t \geq 0 .\right.
$$

Although our new definition of $\tau_{a}$ may look different from the previous one, it is easy to show that the two definitions coincide and that in fact:

Proposition 3.2 (Proof omitted). Let $S_{t}$ be as (3.3). Then for all $t \geq 0$,

$$
S_{t}=\left\{v \in \mathbf{V}: \exists a \in[n], Y_{t}(v)=a\right\}
$$

and

$$
\left|S_{t}\right|=\left|\left\{a \in[n]: Y_{t}(a) \neq \partial\right\}\right| .
$$

Therefore, for all $k \in \mathbb{N} \backslash\{0\}$,

$$
\mathbb{P}_{x^{(n)}}\left(C_{k}>t\right)=\mathbb{P}_{x^{(n)}}\left(\left|S_{t}\right| \geq k+1\right)=\mathbb{P}_{x^{(n)}}\left(\left|\left\{a \in[n]: Y_{t}(a) \neq \partial\right\}\right| \geq k+1\right) .
$$

Remark 3.3. As in Remark 3.1, we may allow $x^{(n)}$, where $x(i)=x(j)$ for some pair $i \neq j$. Notice, however, that $Y_{0}^{(n)} \neq x^{(n)}$ in this case.

3.4. Random walks with a list of allowed killings. Now assume that we have a deterministic càdlàg trajectory:

$$
\mathcal{A}: t \geq 0 \mapsto \mathcal{A}_{t} \in 2^{[n]^{2}} .
$$

We define yet another process,

$$
\left(\left(Y_{t}^{\mathcal{A}}\right)^{(n)}\right)_{t \geq 0} \equiv\left(Y_{t}^{\mathcal{A}}(a): a \in[n]\right)_{t \geq 0}
$$

where a walker with index $a$ may be killed by a walker with index $b$ only if they occupy the same site at some time $t$ and $(b, a) \in \mathcal{A}_{t}$. Intuitively, this means that $b$ is allowed to kill $a$ only at times $t$ with $(b, a) \in \mathcal{A}_{t}$.

For a formal definition, we first set

$$
Y_{t}^{\mathcal{A}}(1) \equiv X_{t}(1) \quad(t \geq 0)
$$

Given $a \in[n] \backslash\{1\}$, assume inductively that $\left(Y_{t}^{\mathcal{A}}(b)\right)_{t \geq 0}$ has been defined for $1 \leq$ $b<a$. Define

$$
\tau_{a}^{\mathcal{A}} \equiv \inf \left\{t \geq 0: \exists 1 \leq b<a,(b, a) \in \mathcal{A}_{t} \text { and } X_{t}(a)=Y_{t}^{\mathcal{A}}(b)\right\}
$$


and set

$$
Y_{t}^{\mathcal{A}}(a) \equiv\left\{\begin{array}{ll}
X_{t}(a), & 0 \leq t<\tau_{a}^{\mathcal{A}}, \\
\partial, & t \geq \tau_{a}^{\mathcal{A}},
\end{array} \quad \text { for each } t \geq 0\right.
$$

The following proposition shows that the process with a list of allowed killings can be used to upper bound $\mathbb{E}_{x^{(n)}}\left[C_{k}\right]$.

Proposition 3.4. Define

$$
S_{t}^{\mathcal{A}} \equiv\left\{Y_{t}^{\mathcal{A}}(a): a \in[n]\right\}
$$

For any choice of $\mathcal{A}$ as above and of initial state $x^{(n)}$, one can couple $\left(S_{t}\right)_{t \geq 0}$ and $\left(S_{t}^{\mathcal{A}}\right)_{t \geq 0}$ such that (almost surely) $S_{t} \subset S_{t}^{\mathcal{A}}$ for all $t \geq 0$. In particular, for all $k \in \mathbb{N} \backslash\{0\}$,

$$
\mathbb{P}_{x^{(n)}}\left(C_{k}>t\right)=\mathbb{P}_{x^{(n)}}\left(\left|S_{t}\right| \geq k+1\right) \leq \mathbb{P}_{x^{(n)}}\left(\left|S_{t}^{\mathcal{A}}\right| \geq k+1\right) .
$$

We omit the proof of this rather intuitive proposition. The key idea here is this: suppose we do not kill a walker $a$ at a given time $t_{0}$. The only way this could make $S_{t}$ "smaller" is if $X_{t}(a)$ were to meet a walker $X_{t}(c)$ with $c>a$ at some later time $t \geq t_{0}$. But if this happens, we may pretend that $X_{s}(a)$ follows the trajectory of $X_{s}(c)$ for $s \geq t$. This follows from the Markov property coupled with the fact that $X_{t}(a)=X_{t}(c)$. This shows that in fact $S_{t}$ does not become smaller.

Remark 3.5. Similar to Remark 3.3. we note that we may allow $x^{(n)}$ with $x(i)=x(j)$ for some pair $i \neq j$, but then $\left(Y_{0}^{\mathcal{A}}\right)^{(n)} \neq x^{(n)}$.

\section{Proofs of the main theorems}

We prove Theorems 1.3 and 1.5 in this section. for simplicity, we first focus on the proof of Theorem 1.3 and then show how it can be modified to prove the second theorem. We will take the notation and definitions in Sections 2.2] and 3 for granted.

4.1. Preliminaries for the proof of Theorem 1.3. We first note that Theorem 1.3 follows from a seemingly different statement.

Proposition 4.1. Let $c, \gamma>0$ be given universal constants. Suppose we can show that there exists some choice of $\mathcal{A}=\left(\mathcal{A}_{t}\right)_{t \geq 0}$ as in Section 3.4 and of $0 \leq t_{0} \leq$ $c\left(\mathrm{~T}_{\text {mix }}^{Q}+\mathrm{T}_{\text {hit }}^{Q}\right)$ with

$$
\forall n \in \mathbb{N} \backslash\{0\}, \forall x^{(n)} \in \mathbf{V}^{n}, \mathbb{P}_{x^{(n)}}\left(\left|S_{t_{0}}^{\mathcal{A}}\right| \geq 2\right) \leq 1-\gamma .
$$

Then

$$
\forall n \in \mathbb{N} \backslash\{0\}, \forall x^{(n)} \in \mathbf{V}^{n}, \mathbb{E}_{x^{(n)}}[C] \leq K \mathrm{~T}_{\text {hit }}^{Q},
$$

where $K>0$ is universal.

Proof. Given $s \geq 0$, denote

$$
E(s) \equiv\{C>s\}=\left\{\left|S_{s}\right| \geq 2\right\}=\bigcup_{a \in[n] \backslash\{1\}}\left\{\tau_{a}>s\right\} .
$$

Combining the assumption of the proposition with Proposition 3.4 gives

$$
\forall n \in \mathbb{N} \backslash\{1\}, \forall x^{(n)} \in \mathbf{V}^{n}, \mathbb{P}_{x^{(n)}}\left(E\left(t_{0}\right)\right) \leq \mathbb{P}_{x^{(n)}}\left(\left|S_{t_{0}}^{\mathcal{A}}\right| \geq 2\right) \leq 1-\gamma
$$


We now consider $E\left(\ell t_{0}\right)$, where $\ell>1$ is an integer. Let $\left(\Theta_{s}\right)_{s \geq 0}$ denote the timeshift operators for the independent random walks process and let $\left(\mathcal{F}_{s}^{(n)}\right)_{s \geq 0}$ denote the filtration generated by this process:

$$
\begin{aligned}
\mathbb{P}_{x^{(n)}}\left(E\left(k t_{0}\right)\right) & \leq \mathbb{P}_{x^{(n)}}\left(E\left((\ell-1) t_{0}\right) \cap\left(\bigcup_{a=2}^{n}\left\{\tau_{a} \circ \Theta_{(\ell-1) t_{0}}>t_{0}\right\}\right)\right) \\
& =\mathbb{P}_{x^{(n)}}\left(E\left((\ell-1) t_{0}\right) \cap \Theta_{(\ell-1) t_{0}}^{-1}\left(E\left(t_{0}\right)\right)\right), \\
\left(E\left((\ell-1) t_{0}\right) \in \mathcal{F}_{(\ell-1) t_{0}}^{(n)}\right) & \leq \mathbb{E}_{x^{(n)}}\left[\mathbb{I}_{E\left((\ell-1) t_{0}\right)} \mathbb{P}_{x^{(n)}}\left(\Theta_{(\ell-1) t_{0}}^{-1}\left(E\left(t_{0}\right)\right) \mid \mathcal{F}_{(\ell-1) t_{0}}^{(n)}\right)\right], \\
(\text { Markov property) } & =\mathbb{E}_{x^{(n)}}\left[\mathbb{I}_{E\left((\ell-1) t_{0}\right)} \mathbb{P}_{X_{(\ell-1) t_{0}}^{(n)}}\left(E\left(t_{0}\right)\right)\right], \\
\text { (inequality (4.2) }) & \leq \mathbb{E}_{x^{(n)}}\left[\mathbb{I}_{E\left((\ell-1) t_{0}\right)}(1-\gamma)\right] \\
& =\mathbb{P}_{x^{(n)}}\left(E\left((\ell-1) t_{0}\right)\right)(1-\gamma), \\
\text { (induction on } \ell) & \leq(1-\gamma)^{\ell} .
\end{aligned}
$$

Recalling the definition of $E(s)$, we deduce that

$$
\forall n \in \mathbb{N} \backslash\{1\}, \forall x^{(n)} \in \mathbf{V}^{n}, \mathbb{E}_{x^{(n)}}[C] \leq \sum_{\ell \in \mathbb{N}}(1-\gamma)^{\ell} t_{0}=\frac{t_{0}}{\gamma} \leq \frac{c\left(\mathrm{~T}_{\text {mix }}^{Q}+\mathrm{T}_{\text {hit }}^{Q}\right)}{\gamma} .
$$

The proposition follows from this because $\mathrm{T}_{\text {mix }}^{Q} \leq c_{0} \mathrm{~T}_{\text {hit }}^{Q}$ for some universal $c_{0}>0$ [2, Chapter 3] and both $c$ and $\gamma$ are universal.

\subsection{Construction of $\mathcal{A}$.}

Notational convention 4.2. From now on, we fix some $x^{(n)}$ and write $\mathbb{P}$ instead of $\mathbb{P}_{x^{(n)}}$.

We will now design a specific trajectory $\mathcal{A}=\left(\mathcal{A}_{t}\right)_{t \geq 0}$ which will allow for a simple analysis of $S_{t}^{\mathcal{A}}$. Let $m \in \mathbb{N}$ be the smallest non-negative number with $n \leq \sum_{i=0}^{m} 2^{i}$. Define sets

$$
\begin{aligned}
A_{0} & =\{1\} \\
A_{j} & \equiv\left[\sum_{i=0}^{j} 2^{i}\right] \backslash\left[\sum_{i=0}^{j-1} 2^{i}\right](1 \leq j \leq m-1), \\
\text { and } A_{m} & \equiv[n] \backslash\left[\sum_{i=0}^{m-1} 2^{i}\right] .
\end{aligned}
$$

We will consider different epochs, numbered backwards in time. It is convenient to have the following notation:

$$
\begin{aligned}
& t_{m} \equiv 2 \mathrm{~T}_{\text {mix }}^{Q}, \\
& t_{j}=t_{j+1}+(\ln 5) 2^{4-j} \mathrm{~T}_{\text {hit }}^{Q}, \quad j=m-1, m-2, \ldots, 0 .
\end{aligned}
$$

(1) Epoch $\# \infty$ is the time interval $\left[0, t_{m}\right)$. We set $\mathcal{A}_{t} \equiv \emptyset$ for all $t$ in this interval, i.e. no killings are allowed up to time $2 \mathrm{~T}_{\text {mix }}^{Q}$. 
(2) Epochs $\# m$ through \#1 correspond to time intervals $I_{j}=\left[t_{j}, t_{j-1}\right)$ as $j$ decreases from $m$ to 1 . For each such $j$ we set

$$
\mathcal{A}_{t} \equiv A_{j-1} \times \bigcup_{p=j}^{m} A_{j}, t \in I_{j} .
$$

That is, the only killings allowed are between walkers with labels in $A_{j-1}$ and $A_{p}$ with $p \geq j$.

(3) Epoch \#0 corresponds to the time interval

$$
I_{0} \equiv\left[t_{0},+\infty\right)
$$

(the remaining time), where we set $\mathcal{A}_{t} \equiv[n]^{2}$.

We note for later convenience that

$$
t_{0} \leq 2 \mathrm{~T}_{\text {mix }}^{Q}+(\ln 5) \sum_{j \geq 0} 2^{4-j} \mathrm{~T}_{\text {hit }}^{Q} \leq c\left(\mathrm{~T}_{\text {mix }}^{Q}+\mathrm{T}_{\text {hit }}^{Q}\right)
$$

with $c>0$ universal, since $\sum_{j} 2^{-j}<+\infty$. We will use this in our application of Proposition 4.1

4.3. Abundance of good walkers. We have the following simple proposition about the epoch $\# \infty$. Intuitively, it says that, at the end of this epoch, a positive proportion of the random walkers are "good", in that they have converged to stationarity.

Proposition 4.3. One can construct a (random) subset $R \subset[n]$ such that:

(1) $R$ is $\mathcal{H}_{t_{m}}^{(n)}$-measurable, where $\mathcal{H}_{t_{m}}^{(n)}$ is the sigma-field generated by $\left(X_{s}^{(n)}\right)_{s \leq t_{m}}$ and by some additional independent random variable $U$.

(2) Each $r \in[n]$ belongs to $R$ with probability $1 / 4$, independently of all other $r^{\prime} \in[n]$.

(3) Conditionally on $R$ and on $\left(X_{t_{m}}(i)\right)_{i \in[n] \backslash R}$, the vector $\left(X_{t_{m}}(r)\right)_{r \in R}$ has iid coordinates, each with distribution $\pi$.

Proof. Consider a single $a \in[n]$. Since $Q$ is reversible, Lemma 7 in [2, Chapter 4] shows that

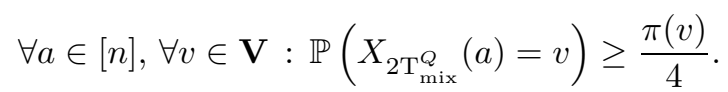

In other words, for each $a$ there exists some $\nu_{a} \in M_{1}(\mathbf{V})$ such that

$$
\mathbb{P}\left(X_{2 \mathrm{~T}_{\text {mix }}^{Q}}(a)=\cdot\right)=\frac{1}{4} \pi(\cdot)+\frac{3}{4} \nu_{a}(\cdot) .
$$

Since the random variables $\left(X_{t_{m}}(a)\right)_{a \in[n]}$ are independent, we may assume that they sampled as follows:

(1) Let $(I(a))_{a \in[n] \backslash A_{m}}$ be iid with $\mathbb{P}(I(a)=1)=1-\mathbb{P}(I(a)=0)=1 / 4$.

(2) For each $a$ with $I(a)=1$, let $X_{t_{m}}(a)$ be a sample from $\pi$, independent of everything else.

(3) For each $b$ with $I(b)=0$, let $X_{t_{m}}(b)$ be a sample from $\nu_{b}$, independent of everything else.

One may check that $R \equiv\left\{a \in[n] \backslash A_{m}: I(a)=1\right\}$ has the desired properties.

The next proposition means that, with positive probability, there is a constant proportion of good walkers within each $A_{i}$ with $i \leq m-1$. 
Proposition 4.4. Let $\mathcal{G}$ be the event

$$
\mathcal{G} \equiv \bigcap_{i=0}^{m-1}\left\{\left|R \cap A_{i}\right| \geq 2^{i-3}\right\} .
$$

Then $\mathbb{P}(\mathcal{G}) \geq \alpha>0$, where

$$
\alpha \equiv \prod_{i=0}^{+\infty}\left(1-e^{-2^{i-7}}\right)>0
$$

is universal.

Proof. Let $\operatorname{Bin}(m, x)$ denote a binomial random variable with parameters $m$ and $x$ so that

$$
\mathbb{P}(\operatorname{Bin}(m, x)=k)=\left(\begin{array}{c}
m \\
k
\end{array}\right) x^{k}(1-x)^{m-k} \quad(k \in[m] \cup\{0\}) .
$$

The random variables $\left|R \cap A_{i}\right|, 0 \leq i \leq m-1$, are independent, and each has the law of $\operatorname{Bin}\left(2^{i}, 1 / 4\right)$ :

$$
\mathbb{P}\left(\left|R \cap A_{i}\right|<2^{i-3}\right)=\mathbb{P}\left(\operatorname{Bin}\left(2^{i}, 1 / 4\right)<\mathbb{E}\left[\operatorname{Bin}\left(2^{i}, 1 / 4\right)\right]-2^{i-3}\right) .
$$

Chernoff bounds [3, Appendix A.1] imply that

$$
\mathbb{P}\left(\left|R \cap A_{i}\right|<2^{i-3}\right) \leq e^{-\frac{\left(2^{i-3}\right)^{2}}{2.2^{i}}}=e^{-2^{i-7}} .
$$

We deduce that

$$
\mathbb{P}(\mathcal{G}) \geq \prod_{i=0}^{m-1} \mathbb{P}\left(\operatorname{Bin}\left(2^{i}, 1 / 4\right) \geq 2^{i-3}\right) \geq \alpha .
$$

The positivity of $\alpha$ follows from $0<e^{-2^{i-7}}<1$ for all $i$ and $\sum_{i} e^{-2^{i-7}}<+\infty$.

4.4. The probability of being alive. Let $E(a, t)$ denote the event

$$
E(a, t) \equiv\left\{Y_{t}^{\mathcal{A}}(a) \neq \partial\right\}=\left\{\tau_{a}^{\mathcal{A}}>t\right\} .
$$

Notice that

$$
\left\{\left|S_{t}^{\mathcal{A}}\right| \geq 2\right\} \subset \bigcup_{a=2}^{n} E(a, t) .
$$

We now estimate the conditional probability of $E(a, t)$ given $\mathcal{G}$.

Proposition 4.5. Let $a \in A_{i}$ for some $1 \leq i \leq m$. Then for all $1 \leq j<i$,

$$
\mathbb{P}\left(E\left(a, t_{j}\right) \mid \mathcal{G}\right) \leq 5^{j-i} .
$$

Proof. We will prove a stronger statement that for almost all $R_{0} \subset[n]$ and $h=$ $\left(h_{t}\right)_{t \in\left[t_{i}, t_{j}\right)}$,

$$
\mathbb{P}\left(E\left(a, t_{j}\right) \mid R=R_{0},\left(X_{t}(a)\right)_{t \in\left[t_{i}, t_{j}\right)}=\left(h_{t}\right)_{t \in\left[t_{i}, t_{j}\right)}\right) \leq 5^{-\sum_{r=j}^{i-1} \frac{\left|R_{0} \cap A_{r}\right|}{2^{r-4}}} .
$$

This implies the proposition because the occurrence of $\mathcal{G}$ implies $\left|R \cap A_{r}\right| \geq 2^{r-4}$ for all $1 \leq r<m-1$. To avoid notational clutter, we will fix $R_{0}$ and $h$ from now on and will use the following shorthand: for any event $U$,

$$
\widetilde{\mathbb{P}}(U) \equiv \mathbb{P}\left(U \mid R=R_{0},\left(X_{t}(a)\right)_{t \in\left[t_{i}, t_{j}\right)}=\left(h_{t}\right)_{t \in\left[t_{i}, t_{j}\right)}\right) .
$$


To prove (4.6) we first observe that the event $E\left(a, t_{j}\right)$ satisfies:

Claim 4.6. Suppose $b \in A_{r}$ with $j \leq r<i$. Then

$$
E\left(a, t_{j}\right) \subset\left\{\forall t \in\left[t_{r+1}, t_{r}\right), X_{t}(a) \neq X_{t}(b)\right\} .
$$

Proof of the claim. If the event on the RHS does not hold, there exists a $t \in$ $\left[t_{r}, t_{r-1}\right)$ with $X_{t}(a)=X_{t}(b)$. We now argue that $\tau_{a}^{\mathcal{A}} \leq t$ in this case. Indeed, this follows from the definition of $\tau_{a}^{\mathcal{A}}$ and the following observations:

(1) $X_{t}(a)=Y_{t}^{\mathcal{A}}(b)$ : this follows from $X_{t}(b)=Y_{t}^{\mathcal{A}}(b)$, which is a consequence of the fact that $(c, b) \notin \mathcal{A}_{s}$ for any $c<b$ and $s \leq t_{r}$ (i.e. $b$ cannot be killed before time $\left.t_{r}\right)$.

(2) $(b, a) \in \mathcal{A}_{t}$ : this follows from $t \in\left[t_{r+1}, t_{r}\right)=I_{r+1}$.

The claim implies

$$
\begin{aligned}
\widetilde{\mathbb{P}}\left(E\left(a, t_{j}\right)\right) & \leq \widetilde{\mathbb{P}}\left(\bigcap_{r=j}^{i-1} \bigcap_{b \in A_{r}}\left\{\forall t \in\left[t_{r}, t_{r-1}\right), X_{t}(a) \neq X_{t}(b)\right\}\right) \\
& \leq \widetilde{\mathbb{P}}\left(\bigcap_{r=j}^{i-1} \bigcap_{b \in A_{r} \cap R_{0}}\left\{\forall t \in\left[t_{r+1}, t_{r}\right), X_{t}(a) \neq X_{t}(b)\right\}\right) .
\end{aligned}
$$

Now recall from (4.7) that $\widetilde{\mathbb{P}}(\cdot)$ is a conditional probability given $R=R_{0}$ and the trajectory of $X_{s}(a)$. Since $a \notin \bigcup_{r=j}^{i-1} A_{r}$, Proposition 4.3 implies that

Under $\widetilde{\mathbb{P}}(\cdot),\left(X_{t_{m}}(b): b \in R_{0} \cap\left(\bigcup_{r=j}^{i-1} A_{r}\right)\right)$ are iid with common law $\pi$.

Since $R$ is $\mathcal{H}_{t_{m}}^{(n)}$-measurable, the Markov property for the independent random walks process leads to the following conclusion:

Under $\widetilde{\mathbb{P}}(\cdot),\left(X_{t+t_{m}}(b): b \in R_{0} \cap\left(\bigcup_{r=j}^{i-1} A_{r}\right)\right)_{t \geq 0}$ are iid realizations of $\mathbb{P}_{\pi}$.

We deduce that

$$
\begin{aligned}
\widetilde{\mathbb{P}}\left(E\left(a, t_{j}\right)\right) & =\prod_{r=j}^{i-1} \prod_{b \in R_{0} \cap A_{r}} \mathbb{P}_{\pi}\left(\forall t \in\left[t_{r+1}, t_{r}\right), X_{t} \neq h_{t}\right), \\
\left(\mathbb{P}_{\pi} \text { stationary }\right) & =\prod_{r=j}^{i-1} \mathbb{P}_{\pi}\left(\forall t \in\left[0, t_{r}-t_{r+1}\right), X_{t}(b) \neq h_{t+t_{r}}\right)^{\left|R_{0} \cap A_{r}\right|} .
\end{aligned}
$$

We apply the Meeting Time Lemma (Lemma 1.7 above) to each term in the product and deduce that, for some choice of $\left(v, \mathbf{q}_{v}\right)$ as in the lemma,

$$
\widetilde{\mathbb{P}}\left(E\left(a, t_{j}\right)\right) \leq \prod_{r=j}^{i-1}\left\{\exp \left(-\frac{\left(t_{r}-t_{r+1}\right)\left|R_{0} \cap A_{r}\right|}{\mathbb{E}_{\mathbf{q}_{v}}\left[H_{v}\right]}\right)\right\} .
$$

The proof of (4.6) finishes once we realize that $t_{r}-t_{r+1}=2^{4-r}(\ln 5) \mathrm{T}_{\text {hit }}^{Q}$ and $\mathrm{T}_{\text {hit }}^{Q} \geq \mathbb{E}_{\mathbf{q}_{v}}\left[H_{v}\right]$. 
4.5. End of proof of Theorem 1.3. We now complete the proof of Theorem 1.3 By Proposition 4.1, it suffices to show that

$$
\mathbb{P}_{x^{(n)}}\left(\left|S_{t_{0}}^{\mathcal{A}}\right| \geq 2\right) \leq 1-\gamma
$$

for some universal $\gamma>0$, with $t_{0}$ as in (4.4). To see this, we will use (4.5) and recall our convention of omitting $x^{(n)}$ from the notation (cf. Notational convention 4.2):

$$
\begin{aligned}
\mathbb{P}\left(\left|S_{t_{0}}^{\mathcal{A}}\right| \geq 2\right) & =\mathbb{P}\left(\bigcup_{a=2}^{n} E\left(a, t_{0}\right)\right) \\
(\mathcal{G} \text { as in Proposition 4.4) } & \leq 1-\mathbb{P}(\mathcal{G})+\mathbb{P}\left(\mathcal{G} \cap \bigcup_{a=2}^{n} E\left(a, t_{0}\right)\right), \\
(\text { union bound }) & \leq 1-\mathbb{P}(\mathcal{G})+\sum_{a=2}^{n} \mathbb{P}\left(\mathcal{G} \cap E\left(a, t_{0}\right)\right), \\
\left([n] \backslash\{1\}=\bigcup_{i=1}^{m} A_{i}\right) & \leq 1-\mathbb{P}(\mathcal{G})+\mathbb{P}(\mathcal{G}) \sum_{i=1}^{m} \sum_{a \in A_{i}} \mathbb{P}\left(E\left(a, t_{0}\right) \mid \mathcal{G}\right), \\
\left.(\text { Proposition } 4.5]+\left|A_{i}\right| \leq 2^{i}\right) & \leq 1-\mathbb{P}(\mathcal{G})+\mathbb{P}(\mathcal{G}) \sum_{i=1}^{+\infty}\left(\frac{2}{5}\right)^{i}, \\
& =1-\frac{\mathbb{P}(\mathcal{G})}{3} .
\end{aligned}
$$

Since $\mathbb{P}(\mathcal{G}) \geq \alpha$ for some universal $\alpha>0$ (cf. Proposition 4.4), we deduce that

$$
\mathbb{P}_{x^{(n)}}\left(\left|S_{t_{0}}^{\mathcal{A}}\right| \geq 2\right) \leq 1-\gamma \text { with } \gamma \equiv \frac{\alpha}{3} \text { universal. }
$$

This finishes the proof.

4.6. Proof of Theorem 1.5. We now present the modifications of the previous proof that are necessary to prove Theorem 1.5. We keep the definitions from previous subsections. We will also assume that $k>4$ so that there exists some $j \in[\mathrm{m}]$ with

$$
h \equiv 2^{j+1}-1=1+2+\cdots+2^{j}<k / 2 .
$$

In fact, we will assume that $j$ is the largest number satisfying this so that $2^{j+2} \geq$ $k / 2$. (The case of $k \leq 4$ follows from Theorem 1.3, with an increase in the universal constant if necessary. If $m$ is too small to allow for this choice of $j$, we may increase $n$ - and thus $m$ - at the cost of having more walkers in the beginning of the process.)

We first need an analogue of Proposition 4.1 .

Proposition 4.7 (Proof omitted). Suppose that there exists a universal $\gamma>0$ such that for all $k$ as above, all $n \in \mathbb{N}$ and all $x^{(n)} \in \mathbf{V}^{n}$,

$$
\mathbb{P}_{x^{(n)}}\left(\left|S_{t_{j}}^{\mathcal{A}}\right| \geq k+1\right) \leq 1-\gamma .
$$

Then there exists a universal $K_{1}>0$ with

$$
\mathbb{E}_{x^{(n)}}\left[C_{k}\right] \leq K_{1}\left(\frac{\mathrm{T}_{\text {hit }}^{Q}}{k}+\mathrm{T}_{\text {mix }}^{Q}\right) .
$$


We omit the proof of this, which follows that of Proposition 4.1 quite closely. The key point is to notice that

$$
t_{j}=2 \mathrm{~T}_{\text {mix }}^{Q}+\sum_{i=j}^{m} 2^{4-i}(\ln 5) \mathrm{T}_{\text {hit }}^{Q} \leq 2 \mathrm{~T}_{\text {mix }}^{Q}+c_{1} 2^{-j-1} \mathrm{~T}_{\text {hit }}^{Q} \leq 2 \mathrm{~T}_{\text {mix }}^{Q}+c_{2} \frac{\mathrm{T}_{\text {hit }}^{Q}}{k}
$$

with $c_{1}, c_{2}>1$ universal (here we used $2^{j+2} \geq k / 2$ ).

We will now bound $\mathbb{P}_{x^{(n)}}\left(\left|S_{t_{j}}^{\mathcal{A}}\right| \geq k+1\right)$ in terms of $h$ and $k$. Using Notational convention 4.2, we first observe that, since $h<k$,

$$
\mathbb{P}\left(\left|S_{t_{j}}^{\mathcal{A}}\right| \geq k+1\right)=\mathbb{P}\left(\sum_{a=1}^{n} \mathbb{I}_{E\left(a, t_{j}\right)} \geq k+1\right) \leq \mathbb{P}\left(\sum_{a=h+1}^{n} \mathbb{I}_{E\left(a, t_{j}\right)} \geq k-h+1\right) .
$$

Now follow the long chain of inequalities in the previous subsection to deduce that

$$
\begin{aligned}
\mathbb{P}\left(\left|S_{t_{j}}^{\mathcal{A}}\right| \geq k+1\right) & \leq 1-\mathbb{P}(\mathcal{G})+\mathbb{P}(\mathcal{G}) \mathbb{P}\left(\sum_{a=h+1}^{n} \mathbb{I}_{E\left(a, t_{j}\right)} \geq k-h+1 \mid \mathcal{G}\right), \\
\text { (Markov ineq. }) & \leq 1-\mathbb{P}(\mathcal{G})+\mathbb{P}(\mathcal{G}) \frac{\mathbb{E}\left[\sum_{a=h+1}^{n} \mathbb{I}_{E\left(a, t_{j}\right)} \mid \mathcal{G}\right]}{k-h+1}, \\
\left([n] \backslash[h]=\bigcup_{i=j+1}^{m} A_{i}\right) & \leq 1-\mathbb{P}(\mathcal{G})+\mathbb{P}(\mathcal{G}) \frac{\mathbb{E}\left[\sum_{i=j+1}^{m} \sum_{a \in A_{i}} \mathbb{I}_{E\left(a, t_{j}\right)} \mid \mathcal{G}\right]}{k-h+1}, \\
& =1-\mathbb{P}(\mathcal{G})+\mathbb{P}(\mathcal{G}) \sum_{i=j+1}^{m} \frac{\sum_{a \in A_{i}} \frac{\mathbb{P}\left(E\left(a, t_{j}\right) \mid \mathcal{G}\right)}{k-h+1},}{\left(\text { Prop. } 4.5+\left|A_{i}\right| \leq 2^{i}\right)} \leq 1-\mathbb{P}(\mathcal{G})+\mathbb{P}(\mathcal{G}) \sum_{i=j+1}^{+\infty} \frac{2^{j}\left(\frac{2}{5}\right)}{k-h+1} \\
& \leq 1-\mathbb{P}(\mathcal{G})+\mathbb{P}(\mathcal{G}) \frac{2^{j+1}}{3(k-h+1)} \\
\left(2^{j+1}=h+1\right) & \leq 1-\mathbb{P}(\mathcal{G})+\mathbb{P}(\mathcal{G}) \frac{h+1}{3(k-h+1)}, \\
(h<k / 2) & \leq 1-\mathbb{P}(\mathcal{G})+\mathbb{P}(\mathcal{G}) \frac{k+2}{6(k-k / 2)} \\
& =1-\mathbb{P}(\mathcal{G})+\mathbb{P}(\mathcal{G}) \frac{k+2}{3 k}, \\
(k>4) & \leq 1-\frac{8}{15} \mathbb{P}(\mathcal{G}) .
\end{aligned}
$$

To finish, we note that $\mathbb{P}(\mathcal{G}) \geq \alpha>0$ with $\alpha$ universal (Proposition 4.4); hence we may take $\gamma=8 \alpha / 15$ in Proposition 4.7

\section{On the Meeting Time Lemma}

5.1. Preliminaries on quasistationary distributions. In this section we review some facts about quasistationary distributions that will be needed in the proof of Lemma 1.7. We will use the definitions in Section 2.2 throughout this section. 
Given any $v \in \mathbf{V}, \mathrm{q}_{v} \in M_{1}(\mathbf{V})$ is a quasistationary distribution for $\mathbf{V} \backslash\{v\}$ if

$$
\forall b \in \mathbf{V}, \mathbf{q}_{v}(b)=\mathbb{P}_{\mathbf{q}_{v}}\left(X_{t}=b \mid H_{v}>t\right) .
$$

All quasistationary distributions $\mathbf{q}_{v}$ correspond to eigenvalues of the restriction of $\Pi^{1 / 2} Q \Pi^{-1 / 2}$ to a subspace $\mathbb{R}_{-v}^{\mathbf{V}}$ of $\mathbb{R}^{\mathbf{V}}$ defined below. Here is the recipe.

(1) Consider the subspace

$$
\mathbb{R}_{-v}^{\mathbf{V}} \equiv\left\{u \in \mathbb{R}^{\mathbf{V}}: u(v)=0\right\}
$$

and let $\mathcal{P}_{-v}: \mathbb{R}^{\mathbf{V}} \rightarrow \mathbb{R}_{-v}^{\mathbf{V}}$ denote the standard projection onto $\mathbb{R}_{-v}^{\mathbf{V}}$.

$$
Q_{-v} \equiv \mathcal{P}_{-v} \Pi^{1 / 2} Q \Pi^{-1 / 2} \mathcal{P}_{-v}
$$

is a symmetric linear operator from $\mathbb{R}_{-v}^{\mathbf{V}}$ to itself with identical diagonal entries and non-positive off-diagonal entries in the "obvious" basis for that space, i.e. the one given by the canonical basis vectors $e_{b}, b \in \mathbf{V} \backslash\{v\}$.

(2) By Perron-Frobenius, each irreducible block of the matrix $Q_{-v}$ has a unique eigenvector $w_{v} \in \mathbb{R}_{-v}^{\mathbf{V}} \backslash\{0\}$ with non-negative entries which achieves the smallest eigenvalue $\lambda\left(w_{v}\right)$ corresponding to that block.

(3) A simple calculation shows that the vector

$$
\mathbf{q}_{v} \equiv \frac{\Pi^{1 / 2} w_{v}}{\sum_{b \in \mathbf{V}} \pi^{1 / 2}(b) w_{v}(b)}
$$

defines a probability distribution over $\mathbf{V}$ with

$$
\mathbb{P}_{\mathbf{q}_{v}}\left(X_{t}=b, H_{v}>t\right)=\mathbf{q}_{v}^{\dagger} e^{-t \mathcal{P}_{-v} Q \mathcal{P}_{-v}} e_{b}=e^{-\lambda\left(w_{v}\right) t} \mathbf{q}_{v}(b),
$$

which in particular implies that $\mathbf{q}_{v}$ is a quasistationary distribution associated with $v$. In particular, $\mathbb{E}_{\mathbf{q}_{v}}\left[H_{v}\right]=1 / \lambda\left(w_{v}\right)>0$. Notice moreover that $\mathrm{q}_{v}(v)=0$.

The following proposition - an immediate consequence of the third item above - will be all we need.

Proposition 5.1. Let $Q_{-v}$ be defined as above and let $\lambda(v)$ denote the smallest eigenvalue of $Q_{-v}$. Then there exists a quasistationary distribution $\mathbf{q}_{v}$ for $\mathbf{V} \backslash\{v\}$ such that $\lambda(v)=1 / \mathbb{E}_{\mathbf{q}_{v}}\left[H_{v}\right]$ and

$$
\mathbb{P}_{\mathbf{q}_{v}}\left(H_{v}>t\right)=e^{-\lambda(v) t} .
$$

Proof. This smallest eigenvalue is the smallest eigenvalue of some block of $Q_{-v}$ and thus equals some $w_{v}$. The rest follows from item (3) and from summing the formula for $\mathbb{P}_{\mathrm{q}_{v}}\left(X_{t}=b, H_{v}>t\right)$ over $b$.

\subsection{Proof of the Meeting Time Lemma.}

Proof of Lemma 1.7. Fix $n \in \mathbb{N} \backslash\{0\}, \Delta>0$. We note that

$$
\begin{aligned}
\mathbb{P}_{\pi}\left(\forall 0 \leq s \leq t, X_{s} \neq h_{s}\right) & \leq \mathbb{P}_{\pi}\left(\bigcap_{i=1}^{n}\{X(i t / n) \neq h(i t / n)\}\right) \\
& \leq \mathbb{E}_{\pi}\left[\prod_{i=1}^{n}\left(1+\left(e^{-\frac{\Delta}{n}}-1\right) \mathbb{I}_{\{X(i t / n)=h(i t / n)\}}\right)\right] .
\end{aligned}
$$


For a given $v \in \mathbf{V}$, let $D_{v}$ be the matrix with a 1 in position $(v, v)$ and 0 s elsewhere. A calculation reveals that the RHS above can be rewritten as

$$
(\Pi 1)^{\dagger}\left\{\prod_{1 \leq i \leq n}^{*} \exp \left(-\frac{t Q}{n}\right) \exp \left(-\frac{\Delta D_{h(i t / n)}}{n}\right)\right\} \mathbf{1},
$$

where $\mathbf{1}$ is the all-ones vector, $\Pi=\operatorname{diag}(\pi(v))_{v \in \mathbf{V}}$ was introduced in Section 5.1 and the $\prod^{*}$ symbol means that the order of the terms in the product is from left to right. Since $\Pi$ commutes with all $D_{v}$, we can rewrite the above expression as

$$
\left(\Pi^{1 / 2} \mathbf{1}\right)^{\dagger}\left\{\prod_{1 \leq i \leq n}^{*} \exp \left(-\frac{t \Pi^{1 / 2} Q \Pi^{-1 / 2}}{n}\right) \exp \left(-\frac{\Delta D_{h(i t / n)}}{n}\right)\right\} \Pi^{1 / 2} \mathbf{1} .
$$

The vector $\Pi^{1 / 2} \mathbf{1}$ has norm $\left|\Pi^{1 / 2} \mathbf{1}\right|^{2}=\sum_{v} \pi(v)=1$. This implies that the above expression is at most the operator norm of the product of matrices. It follows that

$$
\mathbb{P}_{\pi}\left(\forall 0 \leq s \leq t, X_{s} \neq h_{s}\right) \leq\left\|\prod_{1 \leq i \leq n}^{*} \exp \left(-\frac{t \Pi^{1 / 2} Q \Pi^{-1 / 2}}{n}\right) \exp \left(-\frac{\Delta D_{h(i t / n)}}{n}\right)\right\|_{\mathrm{op}} .
$$

Since the operator norm is submultiplicative, we obtain

$$
\begin{aligned}
& \mathbb{P}_{\pi}\left(\forall 0 \leq s \leq t, X_{s} \neq h_{s}\right) \\
& \leq \prod_{i=1}^{n}\left\|\exp \left(-\frac{t \Pi^{1 / 2} Q \Pi^{-1 / 2}}{n}\right) \exp \left(-\frac{\Delta D_{h(i t / n)}}{n}\right)\right\|_{\mathrm{op}} \\
& \quad \leq \max _{v \in \mathbf{V}}\left\|\exp \left(-\frac{t \Pi^{1 / 2} Q \Pi^{-1 / 2}}{n}\right) \exp \left(-\frac{\Delta D_{v}}{n}\right)\right\|_{\mathrm{op}}^{n} .
\end{aligned}
$$

We now consider the terms of which we take the maximum in the RHS, for large $n \in \mathbb{N}$. For a given $v \in \mathbf{V}$, we have

$$
\left\|e^{-\frac{t \Pi^{1 / 2} Q \Pi^{-1 / 2}}{n}} e^{-\frac{\Delta D_{v}}{n}}-e^{-\frac{t \Pi^{1 / 2} Q \Pi^{-1 / 2}+\Delta D_{v}}{n}}\right\|_{\text {op }}=O\left(n^{-2}\right),
$$

where the constant implicit in the $O\left(n^{-2}\right)$ term depends on $\Delta, t$ and $Q$, but not on $v$. Letting $n \rightarrow+\infty$ while keeping $\Delta$ fixed, we get

$$
\begin{aligned}
& \lim _{n \rightarrow+\infty}\left(\max _{v \in \mathbf{V}}\left\|e^{-\frac{t \Pi^{1 / 2} Q \Pi^{-1 / 2}}{n}} e^{-\frac{\Delta D_{v}}{n}}\right\|_{\mathrm{op}}\right)^{n} \\
&=\lim _{n \rightarrow+\infty}\left(\max _{v \in \mathbf{V}}\left\|e^{-\frac{t \Pi^{1 / 2} Q \Pi^{-1 / 2}+\Delta D_{v}}{n}}\right\|_{\mathrm{op}}\right)^{n} \\
& \quad=\max _{v \in \mathbf{V}}\left\|e^{-t \Pi^{1 / 2} Q \Pi^{-1 / 2}-\Delta D_{v}}\right\|_{\mathrm{op}} .
\end{aligned}
$$

Indeed, the last line follows from the self-adjointness of the exponential and from the fact that $\left\|B^{k}\right\|_{\mathrm{op}}=\|B\|_{\mathrm{op}}^{k}$ for self-adjoint matrices $B$. We now use the positivedefiniteness of matrix exponentials, together with the spectral mapping property, 
to deduce that

$$
\begin{aligned}
\forall v \in \mathbf{V},\left\|e^{-t \Pi^{1 / 2} Q \Pi^{-1 / 2}-\Delta D_{v}}\right\|_{\mathrm{op}} & =\lambda_{\max }\left(e^{-t \Pi^{1 / 2} Q \Pi^{-1 / 2}-\Delta D_{v}}\right) \\
& =e^{-\lambda_{\min }\left(t \Pi^{1 / 2} Q \Pi^{-1 / 2}+\Delta D_{v}\right)} .
\end{aligned}
$$

The last inequality and (5.2), (5.3) imply that

$$
\mathbb{P}_{\pi}\left(\forall 0 \leq s \leq t, X_{s} \neq h_{s}\right) \leq \exp \left(-\min _{v \in \mathbf{V}, \Delta>0} \lambda_{\min }\left(t \Pi^{1 / 2} Q \Pi^{-1 / 2}+\Delta D_{v}\right)\right) .
$$

We now make the following claim.

Claim 5.2. As $\Delta \nearrow+\infty$,

$$
\lambda_{\min }\left(t \Pi^{1 / 2} Q \Pi^{-1 / 2}+\Delta D_{v}\right) \rightarrow t \lambda_{\min }\left(Q_{-v}\right),
$$

where $Q_{-v}$ is defined as in Section 5.1

This result is probably well known; for instance, it is a weaker variant of Lemma 3.1 in [5. We will prove it below for completeness, but first we deduce from the claim combined with Proposition 5.1 that:

$$
\mathbb{P}_{\pi}\left(\forall 0 \leq s \leq t, X_{s} \neq h_{s}\right) \leq \exp \left(-t \min _{v \in \mathbf{V}} \lambda_{\min }\left(Q_{-v}\right)\right)=e^{-\frac{t}{\mathbb{F}_{q_{v}}\left[H_{v}\right]}}=\mathbb{P}_{\mathbf{q}_{v}}\left(H_{v}>t\right)
$$

where $\mathrm{q}_{v}$ is some quasistationary distribution associated with $v$. This shows that the lemma follows from the claim.

We now prove the claim. Recall the definition of $\mathcal{P}_{-v}$ in Section 5.1 and notice that $D_{v}=I-\mathcal{P}_{-v}$. In particular, $D_{a} w=0$ for all $w \in \mathbb{R}_{-v}^{\mathbf{V}}$ and

$$
\begin{aligned}
\lambda_{\min }\left(t \Pi^{1 / 2} Q \Pi^{-1 / 2}+\Delta D_{v}\right) & =\inf _{w \in \mathbb{R}^{\mathbf{V}},|w|=1} w^{\dagger}\left(t \Pi^{1 / 2} Q \Pi^{-1 / 2}+\Delta D_{v}\right) \\
& \leq \inf _{w \in \mathbb{R}_{-v},|w|=1} w^{\dagger}\left(t \Pi^{1 / 2} Q \Pi^{-1 / 2}+\Delta D_{v}\right) w \\
& =\inf _{w \in \mathbb{R}_{-v},|w|=1} w^{\dagger}\left(t \Pi^{1 / 2} Q \Pi^{-1 / 2}\right) w \\
\left(\text { use } \mathcal{P}_{-v} w=w\right) & =t \inf _{w \in \mathbb{R}_{-v},|w|=1} w^{\dagger}\left(\mathcal{P}_{-v} \Pi^{1 / 2} Q \Pi^{-1 / 2} \mathcal{P}_{-v}\right) w \\
& =t \lambda_{\min }\left(Q_{-a}\right) .
\end{aligned}
$$

To get an opposite inequality, we set $A=t \Pi^{1 / 2} Q \Pi^{-1 / 2}$ for convenience. We first show that there exists some $c>0$ such that for all large enough $\Delta>0$,

$$
A+\Delta D_{v} \succeq \mathcal{P}_{-v} A \mathcal{P}_{-v}-\frac{c \mathcal{P}_{-v}}{\Delta}+\frac{\Delta}{2} D_{v}
$$

where for symmetric matrices $B_{1}, B_{2}$ with the same size, $B_{1} \preceq B_{2}$ means that $B_{2}-B_{1}$ is positive semidefinite. To see this, we use $\mathcal{P}_{-v}+D_{v}=I$ several times 
and notice that for any $x \in \mathbb{R}^{\mathbf{V}}$,

$$
\begin{aligned}
x^{\dagger}\left(A+\Delta D_{v}\right) x= & x^{\dagger} \mathcal{P}_{-v} A \mathcal{P}_{-v} x+x^{\dagger} D_{v} A D_{v} x+2 x^{\dagger}\left(\mathcal{P}_{-v} A D_{v}\right) x \\
& +\Delta\left(x^{\dagger} D_{v} x\right), \\
(\text { Cauchy-Schwartz } \geq \geq & x^{\dagger} \mathcal{P}_{-v} A \mathcal{P}_{-v} x+x^{\dagger} \frac{\Delta D_{v}}{2} x+\left|D_{v} x\right|^{2}\left(\frac{\Delta}{2}-\|A\|_{\mathrm{op}}\right) \\
& -2\|A\|_{\mathrm{op}}\left|\mathcal{P}_{-v} x\right||| D_{v} x \mid, \\
\text { (assume } \left.\Delta>4\|A\|_{\mathrm{op}}\right) \geq & x^{\dagger}\left(\mathcal{P}_{-v} A \mathcal{P}_{-v}+\frac{\Delta D_{v}}{2}\right) x \\
& +\left(\frac{\sqrt{\Delta}\left|D_{v} x\right|}{2}-\frac{2\|A\|_{\mathrm{op}}\left|\mathcal{P}_{-v} x\right|}{\sqrt{\Delta}}\right)^{2}-\frac{4\|A\|_{\mathrm{op}}\left|\mathcal{P}_{-v} x\right|^{2}}{\Delta}, \\
\left(\text { set } c \equiv 4\|A\|_{\mathrm{op}}\right) \geq & x^{\dagger}\left(\mathcal{P}_{-v} A \mathcal{P}_{-v}-\frac{c \mathcal{P}_{-v}}{\Delta}+\frac{\Delta D_{v}}{2}\right) x .
\end{aligned}
$$

This proves (5.5), which implies that

$$
\lambda_{\min }\left(A+\Delta D_{v}\right) \geq \lambda_{\min }\left(\mathcal{P}_{-v} A \mathcal{P}_{-v}-\frac{c \mathcal{P}_{-v}}{\Delta}+\frac{\Delta}{2} D_{v},\right) .
$$

Notice that the matrix on the RHS of (5.6) has $\mathbb{R}_{-v}^{\mathbf{V}}$ as an invarant subspace, which implies that all of its eigenvectors lie in $\mathbb{R}_{-v}^{\mathbf{V}}$ or in its orthogonal complement. It is easy to see that all vectors in the latter space are eigenvectors with eigenvalue $\Delta / 2$. Therefore, for all large enough $\Delta$ the minimal eigenvalue corresponds to a vector in $\mathbb{R}_{-v}^{\mathbf{V}}$. We deduce that for all large $\Delta>0$,

$$
\begin{aligned}
\lambda_{\min }\left(\mathcal{P}_{-v} A \mathcal{P}_{-v}\right. & \left.-\frac{c \mathcal{P}_{-v}}{\Delta}+\frac{\Delta}{4} D_{v}\right) \\
& =\min _{w \in \mathbb{R}_{-v}^{\mathbf{V}},|w|=1} w^{\dagger}\left(\mathcal{P}_{-v} A \mathcal{P}_{-v}-\frac{c \mathcal{P}_{-v}}{\Delta}\right) w=t \lambda_{\min }\left(Q_{-v}\right)-\frac{c}{\Delta}
\end{aligned}
$$

because $w^{\dagger} \mathcal{P}_{-v} A \mathcal{P}_{-v} w=t w^{\dagger} Q_{-v} w$ for all $w$ as above. Together with (5.4) and (5.6), this shows that, when $\Delta>0$ is large enough,

$$
t \lambda_{\min }\left(Q_{-v}\right)-\frac{c}{\Delta} \leq \lambda_{\min }\left(t \Pi^{1 / 2} Q \Pi^{-1 / 2}+\Delta D_{v}\right) \leq t \lambda_{\min }\left(Q_{-v}\right)
$$

and the claim follows when we let $\Delta \nearrow+\infty$.

\section{Final REMARKS}

- Let $\mathrm{T}_{\text {meet }}^{Q}$ denote the maximum expected meeting time of two independent realizations of $Q$. As noted in Remark 1.2 it is not true in general that $\mathbb{E}[C] \leq K_{2} \mathrm{~T}_{\text {meet }}^{Q}$ for some universal $K_{2}>0$ and all $Q$. Can something like this bound be proved for some interesting class of $Q$ ? A more modest question is whether the constants in the two theorems can be improved.

- The Meeting Time Lemma (Lemma 1.7) can be used in the study of a catand-mouse game proposed in [2, Chapter 4, page 17]. Here a cat moves according to a reversible Markov chain $Q$. A mouse chooses its own trajectory $\left(h_{s}\right)_{s \geq 0}$ and an initial distribution for the cat. Aldous and Fill asked if staying put at some special state gives an optimal strategy for the mouse in terms of maximizing $\mathbb{E}[M]$, where $M$ is the meeting time 
of cat and mouse. Lemma 1.7 implies that this is approximately true if $\mathrm{T}_{\text {mix }}^{Q} \ll \max _{v} \mathbb{E}_{\pi}\left[H_{v}\right]$. In fact, some strategy where the mouse stays put nearly maximizes $\mathbb{P}(M>t)$ simultaneously for all $t \geq 0$. We expect to comment on this and related results in an upcoming note.

\section{REFERENCES}

1. David Aldous, Meeting times for independent Markov chains, Stochastic Processes and their Applications 38 (1991), 185-193. MR.1119980 (93m:60142)

2. David Aldous and James Allen Fill, Reversible Markov Chains and Random Walks on Graphs, book draft, http://www.stat.berkeley.edu/ aldous/RWG/book.html.

3. Noga Alon and Joel Spencer, The Probabilistic Method, second ed., Wiley-Interscience Series in Discrete Mathematics, John Wiley and Sons, New York, 2000. MR:1885388 (2003f:60003)

4. J. Theodore Cox, Coalescing random walks and voter model consensus times on the torus in $Z^{d}$, Annals of Probability 17 (1989), 1333-1366. MR.1048930 (91d:60250)

5. Julia Kempe, Alexei Kitaev, and Oded Regev, The complexity of the local Hamiltonian problem, SIAM J. Comput. 35 (2006), no. 5, 1070-1097. MR2217138(2006m:68037)

6. Thomas M. Liggett, Interacting Particle Systems, Grundlehren der Mathematischen Wissenschaften [Fundamental Principles of Mathematical Sciences], vol. 276, Springer-Verlag, 1985. MR776231 (86e:60089)

Departamento de Matemática, Instituto Nacaional de Matemática Pura e Aplicada, Rio de Janeiro, RJ, BraziL 22430-040

E-mail address: rimfo@impa.br 